\title{
Studio del danneggiamento mediante tomografia in luce del sincrotrone: impatto di un cono d'ombra sulla qualità finale delle ricostruzioni
}

\author{
Francesca Cosmi, Salvatore Scozzese \\ Università degli Studi di Trieste, Dipartimento di Ingegneria Meccanica, via A.Valerio10-Trieste; cosmi@units.it
}

Andrea Bernasconi

Politecnico di Milano, Dipartimento di Meccanica, via La Masa 34 - 20156 Milano

\begin{abstract}
RIASSUNTO. Un'accurata osservazione della geometria tridimensionale di cricche e difetti è necessaria per lo studio dei meccanismi alla base del processo di danneggiamento. I metodi convenzionali utilizzati a questo scopo sono distruttivi o non possiedono una sufficiente risoluzione. Le tecniche di imaging che utilizzano la luce di sincrotrone, ed in particolare la microtomografia (micro-CT) a raggi $\mathrm{X}$, invece, uniscono $\mathrm{i}$ vantaggi di una tecnica non distruttiva ad un'elevata risoluzione spaziale e risultano quindi particolarmente interessanti. Un limite all'applicazione di questa tecnica è costituito dalla propensione della cricca a richiudersi una volta rimosso il carico che ha provocato il danneggiamento, superabile attraverso l'impiego di un dispositivo in grado di esercitare un carico di trazione durante l'acquisizione dei dati. Facendo riferimento al set-up sperimentale della linea SYRMEP di Elettra, il sincrotrone di Trieste, e tralasciando per il momento i vincoli legati a pesi e ingombri, è possibile pensare di inserire tra camera di ionizzazione e CCD una macchina per prove di trazione mono-colonna commerciale, in grado di mantenere aperto il difetto per tutta la durata della tomografia. In questo lavoro viene valutato l'impatto di questo vincolo sulla qualità finale delle ricostruzioni.
\end{abstract}

ABSTRACT. Careful observation of three-dimensional geometry of cracks and defects is necessary in order to study the mechanisms underlying the process of damage. Conventional methods used for this purpose are destructive or have an insufficient resolution. However, the imaging techniques using synchrotron radiation, and in particular the X-ray microtomography (micro-CT), are particularly interesting as they are able to combine the advantages of a non-destructive technique with high spatial resolution. A limit in the application of this technique is the propensity of the crack to reclose upon removal of the load that had caused the damage. This obstacle can be overcome through the use of a test rig capable of exerting a tensile load during the data acquisition. With reference to the experimental set-up of the line SYRMEP of Elettra, the synchrotron radiation facility in Trieste, and ignoring, for the moment, the constraints related to weight and size, it is possible to consider the insertion, between the ionization chamber and the CCD camera, of a commercial single-column machine for tensile testing, able to keep the defect open for the duration of a tomography. In this paper the impact of this constraint on the final quality of the reconstructions is estimated.

KEYWORDS. Compositi rinforzati con fibre corte Microtomografia; Danneggiamento.

\section{INTRODUZIONE}

o sviluppo di modelli predittivi del comportamento di un materiale sottoposto a carichi variabili nel tempo è strettamente legato ad una migliore comprensione quantitativa del processo di nascita della cricca ed in particolare ad un'accurata descrizione della geometria del difetto, in grado di evidenziare i processi alla base del 
danneggiamento a diverse scale, partendo dai meccanismi a livello microscopico (bridging, delaminazione, crescita dei vuoti, ecc) [1].

I metodi convenzionali utilizzati a questo scopo sono distruttivi o non possiedono una sufficiente risoluzione (radiografie convenzionali, tecniche ultrasonorografiche).

Le tecniche di imaging che utilizzano la luce di sincrotrone, ed in particolare la microtomografia (micro-CT) a raggi X, invece, uniscono $\mathrm{i}$ vantaggi di una tecnica non distruttiva ad un'elevata risoluzione spaziale e risultano quindi particolarmente interessanti. La tecnica microtomografica essenzialmente consiste nell'acquisire un grande numero di proiezioni radiografiche ottenute a diverse posizioni angolari del campione (collocato su una tavola rotante) rispetto alla sorgente, normalmente fino a coprire una rotazione totale di 180 gradi. Applicando algoritmi specifici (ad esempio quello basato sul metodo della retro-proiezione), dalle proiezioni angolari ottenute si ricostruiscono le diverse sezioni trasversali (slices) che riproducono la struttura del campione. Sovrapponendo una serie di slices è poi possibile ottenere una rappresentazione volumetrica del campione. Rispetto ad una sorgente convenzionale, la possibilità di utilizzare una sorgente di luce di sincrotrone consente di migliorare la qualità dell'immagine, evitando effetti legati all'indurimento del fascio e consentendo misure quantitative sul campione in esame. Inoltre, la possibilità di impiego di tecniche radiografiche in contrasto di fase consente di evidenziare al meglio i bordi delle discontinuità all'interno del campione, contribuendo ad evidenziare proprio le caratteristiche oggetto di indagine.

Alcuni anni fa, un'indagine svolta presso la stessa linea su provini di alluminio soggetti a fatica flesso-torsionale aveva consentito la ricostruzione 3D delle cricche sviluppate in diverse condizioni [2,3]. I principali ostacoli incontrati nello sviluppo della ricerca furono:

1. la composizione del campione, al limite dell'energia disponibile alla linea SYRMEP del sincrotrone di Trieste, Elettra;

2. la risoluzione della CCD, al tempo di circa 10 microns;

3. la propensione della cricca a richiudersi una volta rimosso il carico.

La prima limitazione può essere superata utilizzando materiali, quali i compositi a base polimerica, che offrono caratteristiche di penetrabilità ai raggi X migliori di quelli metallici. La disponibilità di una nuova CCD consente ora di giungere a pochi micron di risoluzione e rende possibile lo sviluppo di questa ricerca, rimuovendo il secondo ostacolo.

Per quanto riguarda il terzo aspetto, però, la propensione della cricca a richiudersi una volta rimosso il carico che ha provocato il danneggiamento risulta ancor più enfatizzata nel caso di compositi a matrice polimerica, e richiede l'impiego di un dispositivo in grado di esercitare un carico di trazione durante l'acquisizione dei dati. Anche se diverse sono le possibilità di realizzare un tale set-up, i vincoli di spazio/peso e la necessità di contenere i costi limitano di molto le soluzioni praticamente percorribili. Inoltre in letteratura si trovano descritti principalmente dispositivi atti ad applicare un carico di compressione [4-10].

Facendo riferimento al set-up sperimentale della linea SYRMEP della Sincrotrone Trieste, e tralasciando per il momento i vincoli legati a pesi e ingombri, è possibile pensare di inserire tra camera di ionizzazione e CCD una macchina per prove di trazione mono-colonna commerciale, in grado di mantenere aperta la cricca per tutta la durata della tomografia (Fig.1).

L'impiego di questo tipo di configurazione, che ha il vantaggio di essere di molto semplice realizzazione, presenta però un inconveniente, costituito dalla presenza dalla colonna che crea un cono d'ombra sul campione e non permette l'acquisizione delle immagini su $180^{\circ}$, come avviene normalmente.

In questo lavoro viene valutato l'impatto di questo vincolo sulla qualità finale delle ricostruzioni.

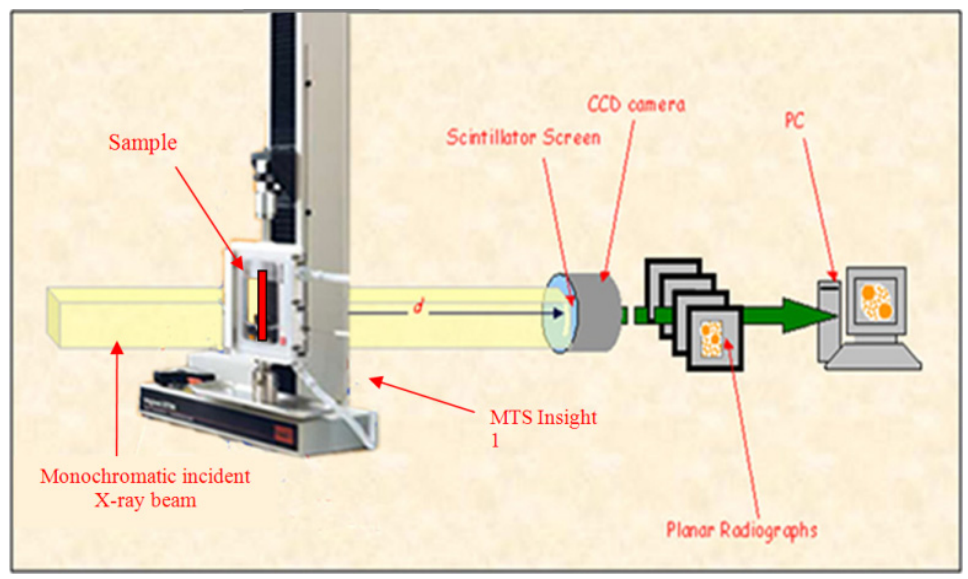

Figura 1: Set-up per micro-CT in condizioni di trazione. 
A partire dai dati di precedenti acquisizioni tomografiche complete, modificando in maniera opportuna il programma normalmente utilizzato per le ricostruzioni, STP (Syrmep Tomo Project), è quindi stata simulata la riduzione del campo visivo dovuta alla presenza della macchina di prova. In particolare, a partire dai due set di dati diversi, sono state eseguite due serie di ricostruzioni, ottenute creando virtualmente un cono d'ombra di dimensioni sempre maggiori, utilizzando via via un numero minore di proiezioni e ricreando così la condizione di acquisizione su angoli inferiori ai $180^{\circ}$.

La qualità delle immagini ottenute e la dimensione massima del cono d'ombra sono state valutate utilizzando i dati provenienti da due tomografie diverse:

- la prima eseguita su un campione in PA6.6 GF35, composito poliammidico rinforzato con fibre corte di vetro;

- la seconda su un campione in Al 5083, proveniente dalla già citata campagna di prove precedenti, [2,3].

\section{CAMPIONE IN PA6.6 GF35}

$\mathrm{L}$

a prima serie di tomografie ottenute con angoli di ricostruzione inferiori a $180^{\circ}$ è stata effettuata su un campione in Poliammide 6.6 rinforzata con il 35\% in peso di fibre corte di vetro (risoluzione 9 micron).

1 Si è proceduto togliendo 5 gradi per volta, rimuovendo quindi di volta in volta dalle ricostruzioni 40 proiezioni per grado, in quanto nella tomografia di partenza erano state acquisite 1440 immagini su $180^{\circ}$, corrispondenti ad un passo angolare di $0.125^{\circ}$. Quindi a $175^{\circ}$ corrisponderanno 1400 immagini, a $170^{\circ}$ corrisponderanno 1360 immagini, e così via togliendo 40 immagini ogni 5 gradi; il tutto mantenendo immutati gli altri parametri di ricostruzione.

I parametri scelti per valutare la bontà delle ricostruzioni fanno riferimento alla possibilità di descrivere la microstruttura assunta dalle fibre di vetro all'interno della matrice polimerica, di cui è possibile una descrizione quantitativa utilizzando il concetto di Mean Intercept Length (MIL) [5, 6, 7]. In questo modo la disposizione delle fibre può essere schematicamente rappresentata mediante un ellissoide, equivalente quindi a un tensore definito positivo del secondo ordine, chiamato MIL fabric tensor, in cui il primo autovettore rappresenta la direzione preferenziale delle fibre e gli autovalori quantificano la tendenza delle fibre ad orientarsi lungo la rispettiva direzione principale (Fig.2).
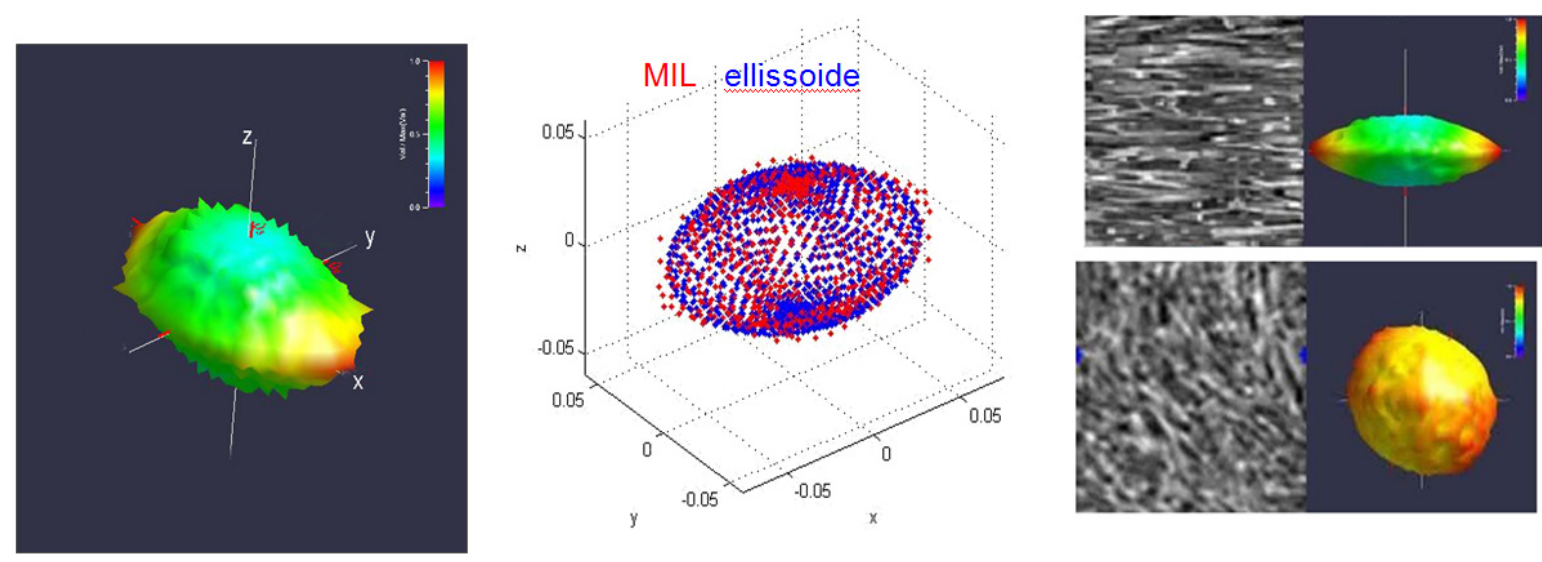

Figura 2: Esempi di rappresentazione del MIL.

Indicando con $\mathrm{H}_{1}, \mathrm{H}_{2}$ e $\mathrm{H}_{3}$ (ordinati dal più grande al più piccolo) gli autovalori, normalizzati in modo che:

$$
\mathrm{H}_{1}+\mathrm{H}_{2}+\mathrm{H}_{3}=1
$$

Si può definire un Indice di anisotropia IA:

$$
\mathrm{IA}=1-\left(\mathrm{H}_{3} / \mathrm{H}_{1}\right)
$$

che vale 0 nel caso di perfetta isotropia e tende a 1 all'aumentare dell'anisotropia.

Come parametri quantitativi per valutare la bontà delle ricostruzioni sono stati quindi utilizzati i valori di IA e del primo autovalore MIL, $\mathrm{H}_{1}$, ottenuti in un volume ricostruito di 80x80x80 voxel3(software Quant3D, parametri come in [11]). I risultati sono riportati in Tab.1.

è stato inoltre effettuato un confronto di tipo visivo su una delle slices, sfruttando la presenza di alcune strutture particolarmente evidenti, che consistono nell'aggregazione di gruppi di fibre. Le immagini ottenute in corrispondenza a diversi angoli di ricostruzione sono riportate in Fig. 3, dove sono evidenziate le zone utilizzate per il confronto visivo. 


\begin{tabular}{ccccc}
\hline $\begin{array}{c}\text { Angolo di } \\
\text { ricostruzione }\end{array}$ & $L A$ & $\% L A$ & $H_{1}$ & $\% H_{1}$ \\
$180^{\circ}$ & 0,48 & - & 0,46 & - \\
$175^{\circ}$ & 0,49 & $2 \%$ & 0,47 & $2 \%$ \\
$170^{\circ}$ & 0,50 & $4 \%$ & 0,48 & $3 \%$ \\
$165^{\circ}$ & 0,51 & $6 \%$ & 0,48 & $4 \%$ \\
$160^{\circ}$ & 0,52 & $8 \%$ & 0,49 & $5 \%$ \\
$155^{\circ}$ & 0,53 & $9 \%$ & 0,50 & $6 \%$ \\
$150^{\circ}$ & 0,53 & $9 \%$ & 0,49 & $6 \%$ \\
$145^{\circ}$ & 0,55 & $12 \%$ & 0,51 & $8 \%$ \\
$140^{\circ}$ & 0,55 & $13 \%$ & 0,51 & $9 \%$ \\
$135^{\circ}$ & 0,55 & $13 \%$ & 0,51 & $9 \%$ \\
$130^{\circ}$ & 0,55 & $12 \%$ & 0,51 & $9 \%$ \\
\hline
\end{tabular}

Tabella 1: Variazione di IA e $\mathrm{H}_{1}$ al diminuire dell'angolo di ricostruzione.

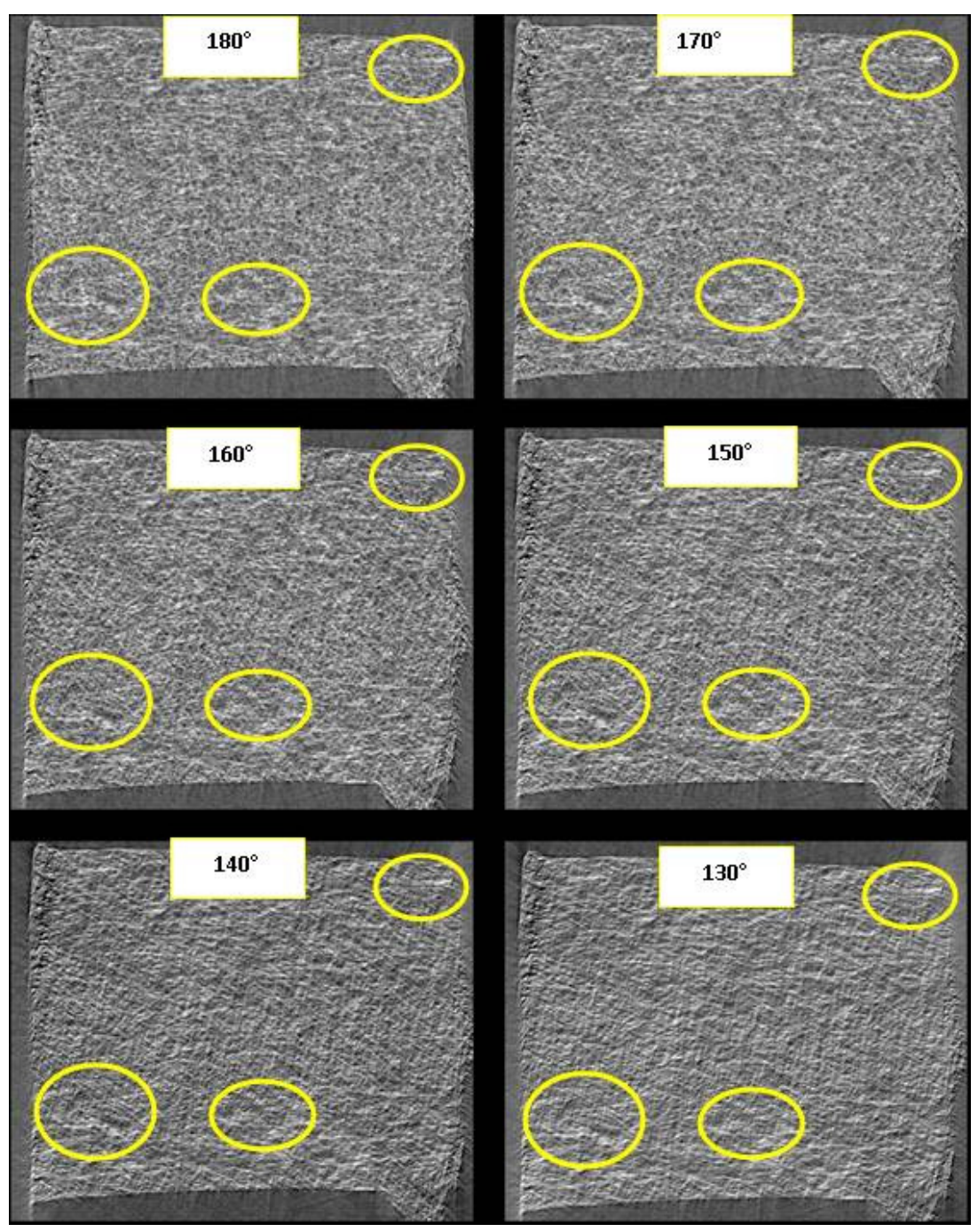

Figura 3 : Confronto visivo al diminuire dell'angolo di ricostruzione di una stessa slice. Le aree evidenziate contengono le strutture utilizzate per il riconoscimento e il confronto delle immagini. 


\section{CAMPIONE IN AL 5083}

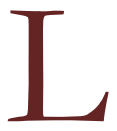

a seconda serie di tomografie ottenute con angoli di ricostruzione inferiori a $180^{\circ}$ è stata effettuata su uno dei campioni studiati in $[2,3]$, in cui venivano presentati i risultati ottenuti nella ricostruzione di cricche di fatica multiassiale utilizzando la tecnica della tomografia in luce di sincrotrone. Il campione qui utilizzato, P8, era stato precedentemente sottoposto presso l'Università di Ferrara a sollecitazione affaticante di flesso-torsione fino al $7 \%$ di caduta di rigidezza, e successivamente tomografato presso la linea SYRMEP del sincrotrone di Trieste (risoluzione 4 micron). La ricostruzione 3D del provino presenta una cricca ben visibile, come osservabile in Fig. 4.

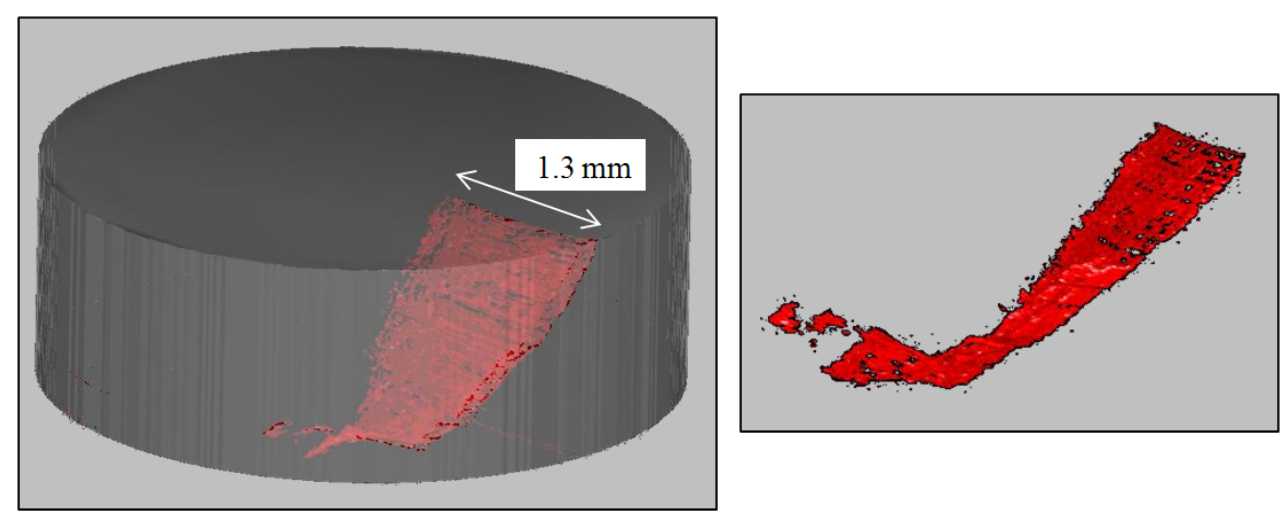

Figura 4: Ricostruzione originale del provino P8 e della cricca (immagine tratta da [3]).

In questo caso la tomografia originale risulta però di qualità inferiore rispetto a quella del campione precedente, essendo stata ottenuta da 360 proiezioni, corrispondenti a 2 immagini per grado di rotazione della tavola porta campione. è stato comunque possibile procedere in modo analogo a quanto fatto per il provino precedente, aumentando via via il cono d'ombra. Sono state considerate solo le slices corrispondenti alla zona in cui è visibile la cricca.

Una volta effettuate le ricostruzioni, i confronti sono stati effettuati:

- $\quad$ su una slice in cui la cricca risultasse ben visibile, come riportato in Fig.5;

- su due viste della cricca, ottenute dopo aver ricostruito tridimensionalmente la cricca mediante il software 3D-

Doctor, come visibile nelle Figg. 6 e 7.

\section{RISULTATI E CONCLUSIONI}

$\mathrm{L}$

e indagini effettuate sul campione in composito rinforzato con fibre corte permettono di affermare che un cono d'ombra di circa $30^{\circ}$ è compatibile con la conservazione delle principali caratteristiche morfologiche, dal momento che lo scarto rispetto al valore dell'indice di anisotropia IA calcolato sulla ricostruzione completa è del $9 \%$ e quello del primo autovalore del MIL di solo $6 \%$. Questo indica che il decadimento della qualità delle ricostruzioni si fa sentire maggiormente nelle direzioni di orientamento delle fibre ortogonali a quella principale, quindi a risentire del cono d'ombra sono maggiormente le direzioni meno importanti dal punto di vista della caratterizzazione del comportamento meccanico del materiale. Inoltre si osserva come le strutture caratteristiche del volume ricostruito (evidenziate in Fig. 3), riconducibili ad aggregazioni di fibre, si mantengano visibili anche per ampiezze del cono d'ombra fino a $30^{\circ}$.

Le immagini della cricca del provino in $\mathrm{Al}$, pur non essendo di qualità paragonabile alla precedente a causa del minor numero di proiezioni utilizzate in partenza, permettono di confermare che la qualità delle ricostruzioni si mantiene accettabile anche in presenza di un cono d'ombra di $30^{\circ}$.

La ricostruzione tomografica con angoli minori di $180^{\circ}$ è dunque una strada percorribile, con un limite massimo di riduzione angolare di circa $30^{\circ}$ per garantire una sufficiente qualità delle immagini.

In commercio sono disponibili numerose macchine per effettuare prove di trazione. In particolare, al fine di mantenere ingombro e peso ridotti, si può pensare di utilizzare macchine che utilizzano celle di carico di capacità limitata, ad esempio 500 o $1000 \mathrm{~N}$, comunque sufficienti a mantenere aperta la cricca durante l'esecuzione della tomografia. Difficilmente però la configurazione standard potrà risultare direttamente utilizzabile per questa applicazione, saranno quindi necessarie delle 
modifiche. Ad esempio, ipotizzando che la colonna sia larga circa $100 \mathrm{~mm}$, sarà necessario posizionare il provino ad almeno $250 \mathrm{~mm}$ dalla colonna stessa, in modo da rispettare i valori desiderati del cono d'ombra.

Un ulteriore problema è poi rappresentato dall'ingombro della macchina per prove di trazione da inserire nel Setup della linea Syrmep, non solo in termini dimensionali, ma anche per quanto riguarda i pesi in quanto è da tener presente che tutta l'apparecchiatura dovrà ruotare con il campione durante tutta la fase di acquisizione. Sarà allora necessario valutare attentamente anche l'aspetto legato al rotore su cui posizionare l'apparecchiatura di prova.
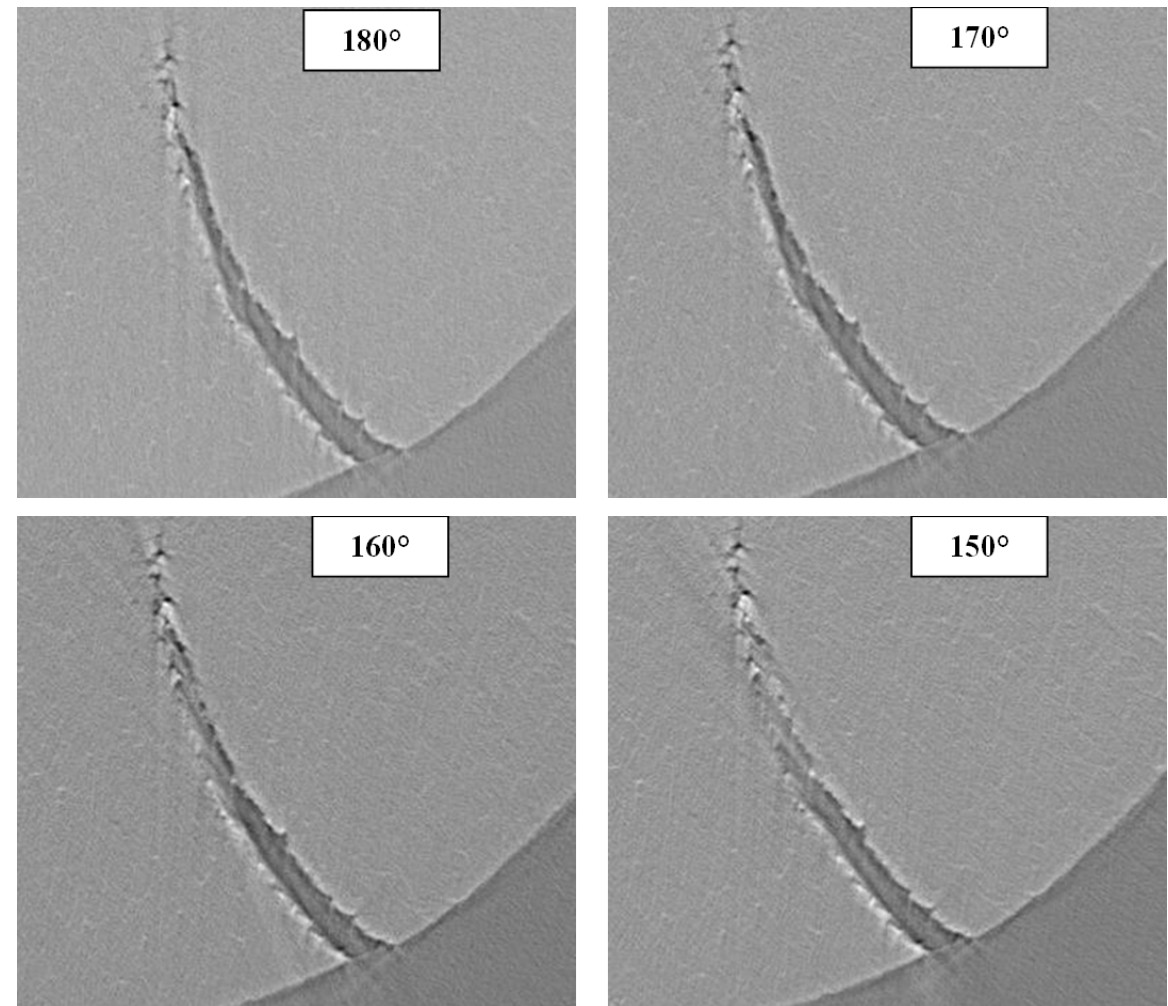

Figura 5: Confronto su una slice al diminuire dell'angolo di ricostruzione della cricca.
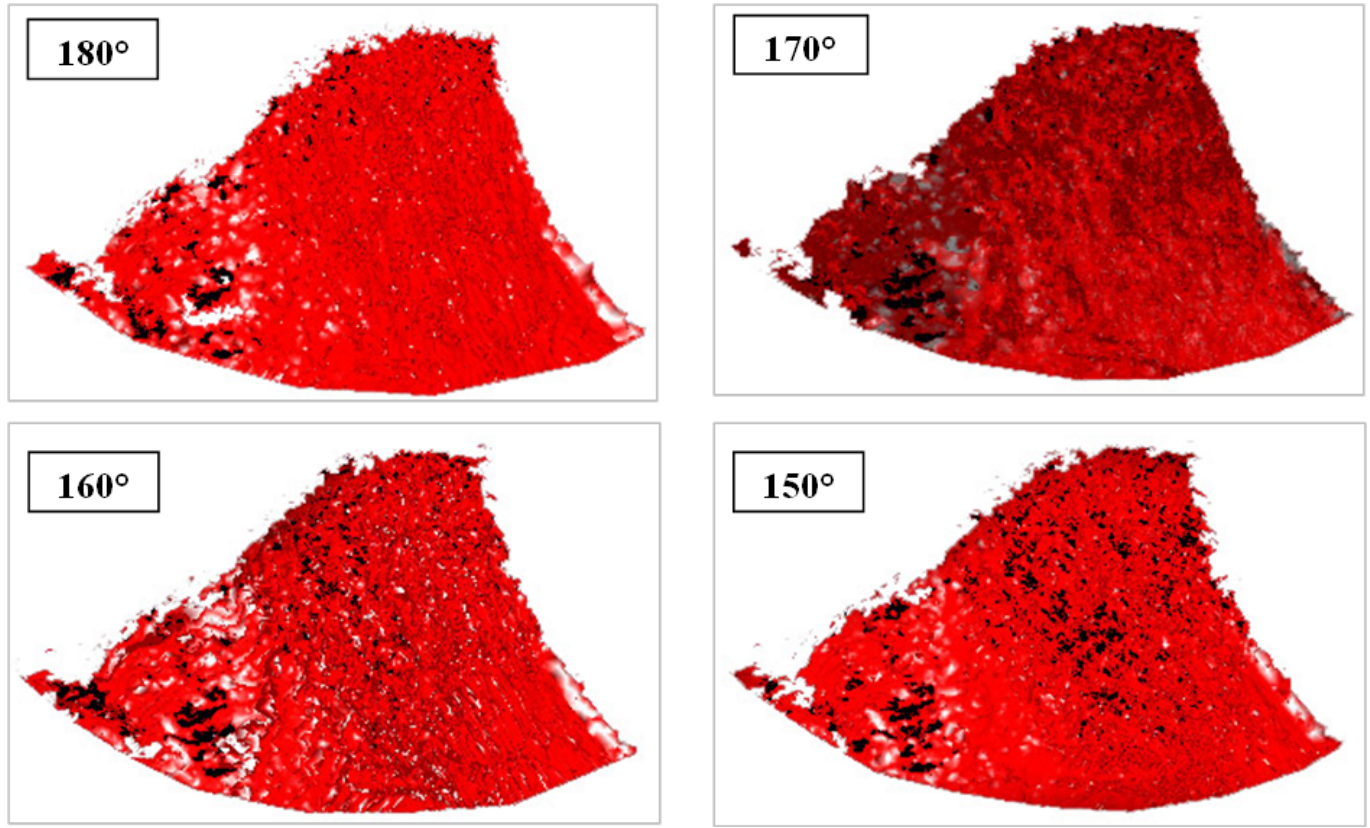

Figura 6: Confronto tra le ricostruzioni 3D della cricca- vista 1. 

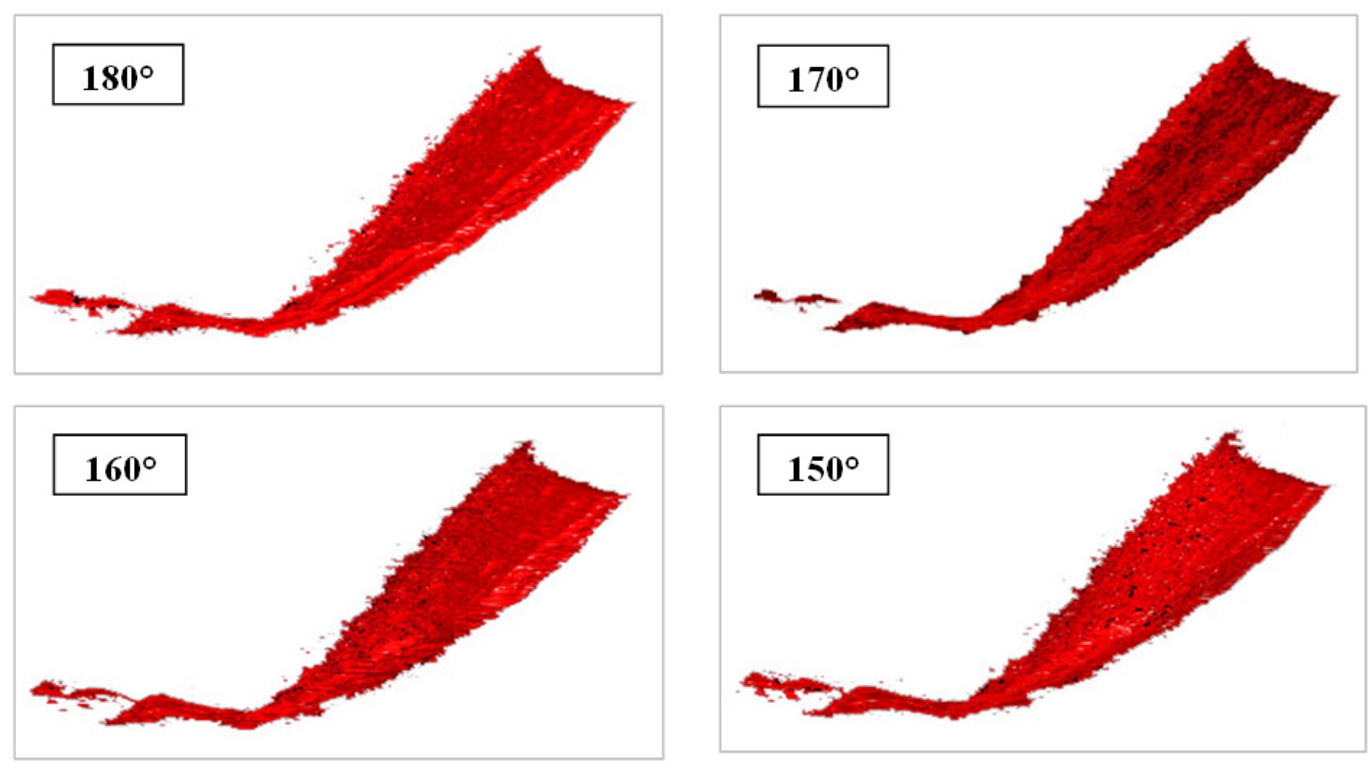

Figura 7: Confronto tra le ricostruzioni 3D della cricca- vista 2.

\section{BIBLIOGRAFIA}

[1] M.E. Launey et al., Acta mater., 57 (2009).

[2] F. Cosmi, A. Cristofori, L. Mancini, R. Tovo, G. Tromba, Proc. of International Conference on Fracture and Damage Mechanics IV, Mallorca, Spagna, (2005).

[3] F. Cosmi, A. Cristofori, L. Mancini, R. Tovo, G. Tromba, M. Volpone, Atti della Gionnata di studio IGF Progettazione a fatica in presenza di multiassialità tensionali, Ferrara, Italia (2005).

[4] J.-Y. Buffiere et al., Acta mater., 47 (5) (1999).

[5] Wang et al., Rev. Sci. Instrum., 76 (2005).

[6] P. Wyss et al., Applications of X-ray Tomographic Microscopy (XTM) in materials and life sciences, EMPA Activities (2002).

[7] M. Rivers, S. Sutton, M. Newville, Workshop on Emerging Scientific Opportunities using X-ray Imaging (2004).

[8] J.-Y. Buffiere et al., ESFR Newsletter (1998)

[9] L. Salvo et al., Nucl. Instr. and Meth. in Phys. Res. B, 200 (2003).

[10] H. Toda et al., Metallurgical and materials Transactions A, 37 (2006).

[11] A. Bernasconi, F. Cosmi, E. Zappa, Strain, Blackwell Publishing, Published Online: DOI: 10.1111/j.14751305.2009.00667.x 